X.-L. YANG, M.-H. XIE, C. ZOU, Y. HE, B. CHEN,* M. O’KEEFFE, C.-D. WU* (ZHEJIANG UNIVERSITY, HANGZHOU, P. R. OF CHINA; UNIVERSITY OF TEXAS AT SAN ANTONIO AND ARIZONA STATE UNIVERSITY, TEMPE, USA)

Porous Metalloporphyrinic Frameworks Constructed from Metal 5,10,15,20-Tetrakis(3,5-biscarboxylphenyl)porphyrin for Highly Efficient and Selective Catalytic Oxidation of Alkylbenzenes

J. Am. Chem. Soc. 2012, 134, 10638-10645.

\section{Porous Metalloporphyrinic Frameworks for Oxidation of Alkylbenzenes}

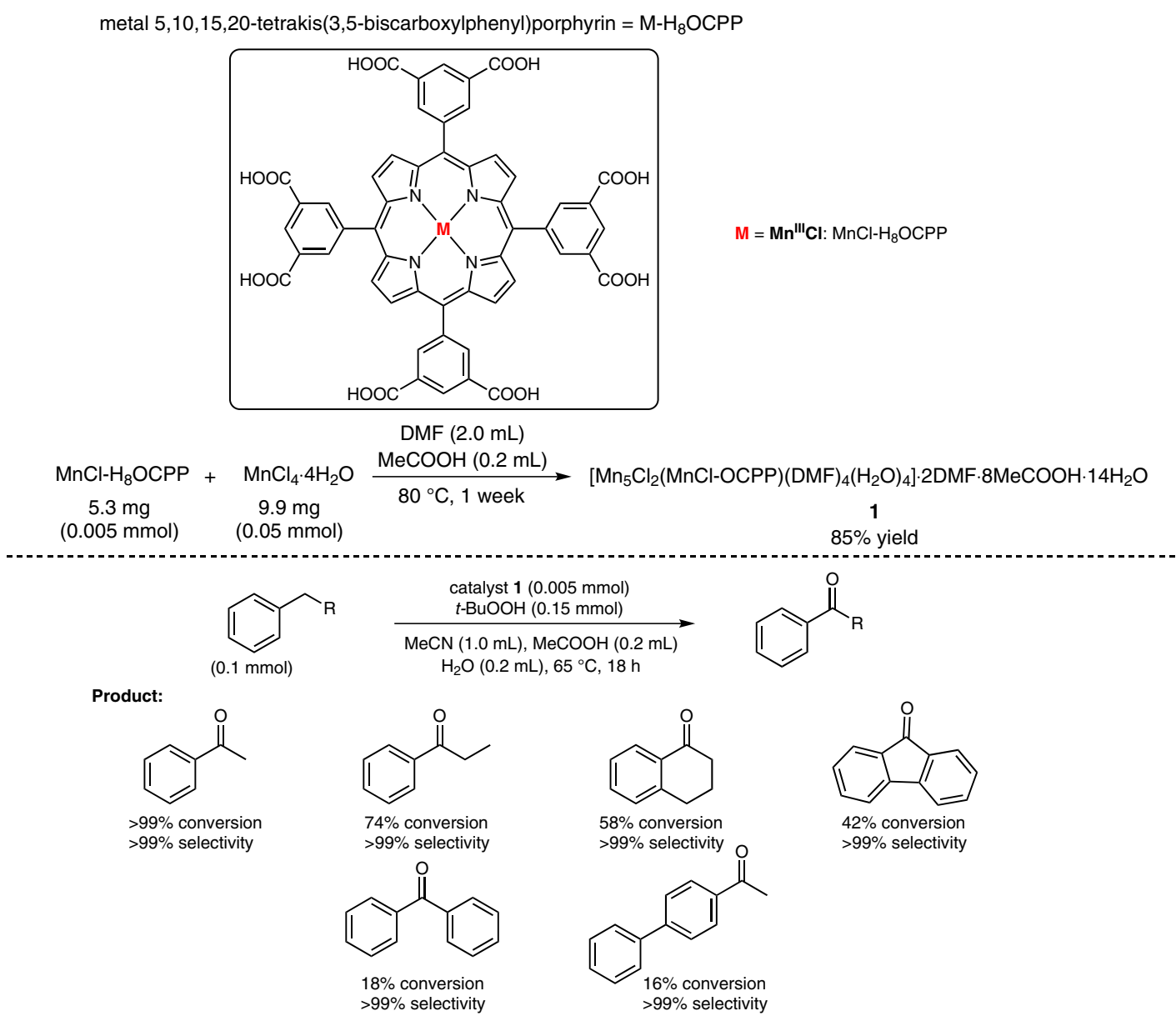

\section{Category}

Polymer-Supported Synthesis

Key words

metalloporphyrins

metal-organic frameworks

manganese(II) chloride

oxidation
Significance: Preparation and characterization of the porous metalloporphyrin octacarboxylate framework 1 are described. The catalytic utility of $\mathbf{1}$ was examined for the oxidation of alkyl benzenes with tert-butyl hydroperoxide as an oxidant to give the corresponding ketones in 16 to $>99 \%$ conversion with $>99 \%$ selectivity (ketones obtained as sole oxidation products from alkylbenzenes).
Comment: The framework 1 was recovered by centrifugation and reused 14 times without significant loss of catalytic activity. A homogeneous metalloporphyrin catalyst $\mathrm{MnCl}-\mathrm{Me}_{8} \mathrm{OCPP}$ showed much lower catalytic activity in the oxidation of ethyl benzene under similar conditions.

SYNFACTS Contributors: Yasuhiro Uozumi, Yoichi M. A. Yamada, Maki Minakawa 